PREDICTORS OF MALE AND FEMALE SERVERS' AVERAGE TIP EARNINGS

\author{
Michael Lynn \& Tony Simons \\ School of Hotel Administration \\ Cornell University
}

In (2000) Journal of Applied Social Psychology, 30, 241-252.

The authors thank Annemarie Schmoyer for help with data collection and data entry for this study. Request reprints from: Michael Lynn, 531 Statler Hall, School of Hotel Administration, Cornell University, Ithaca, NY 14853-6902. 


\begin{abstract}
Tips represent a substantial portion of restaurant waiters' and waitresses' incomes. We report a study that examines several potential predictors of the differences in servers' average tip earnings. Our results indicate that servers earn larger average sales-adjusted tips if they are attractive females, better service providers, and high self-monitors.

However, these effects hold up only for evening tips. None of the variables in this study predicted servers' average lunch tips. The methodological, theoretical, and managerial implications of these findings are discussed.
\end{abstract}




\section{PREDICTORS OF MALE AND FEMALE SERVERS' AVERAGE TIP EARNINGS}

Consumers often give voluntary payments of money (called "tips") to restaurant waiters and waitresses who have served them. In the United States, these tips, which amount to approximately $\$ 12$ billion a year, represent the major source of restaurant servers' incomes (O’Connor, 1971). Since tips are not obligatory and consumers may decide for themselves how much to tip, servers' tip income can vary considerably. For example, one waitress in the present study earned tips that amounted to 17.4 percent of her food and beverage sales while another waitress earned tips that amounted to only 12.1 percent of her sales. A difference between average tips of 12 and 17 percent of sales has enormous income consequences when accumulated over many dining parties, evenings, weeks, etc. Knowledge about the predictors of these differences would inform the employment decisions of restaurant servers and the hiring decisions of restaurant managers. It would also shed light on the psychology underlying consumers' tipping decisions and on the functions of tipping as a social institution.

In this paper, we report on a study designed to increase our knowledge about the predictors of server differences in average tip earnings. The paper is divided into several sections. First, we review relevant theory and research to identify server characteristics that may affect tip earnings. Then, we present the methodology and results of a study testing the relationships between tipping and those server characteristics. Finally, we discuss the methodological, theoretical and practical implications of the study's results. 


\section{LITERATURE REVIEW}

The tipping and social psychology literatures suggest that server differences in average sales-adjusted tips may be attributable to server differences in physical attractiveness, service ability, self-monitoring and sex. These variables and their potential impact on servers' average tips are discussed below.

\section{$\underline{\text { Physical Attractiveness }}$}

Tipping is a norm driven behavior. Consumers tip because they believe that tips are expected and that failure to comply with these expectations brings risks of social disapproval (Crespi, 1947; Lynn \& Grassman, 1990). Research on interpersonal attraction and compliance suggests that people value the approval of attractive individuals more than that of less attractive individuals (see Patzer, 1985), so consumers may tip attractive servers more than unattractive ones. Hornik (1993) and May (1978) have found support for this expectation, but their studies used relatively few servers to operationalize server attractiveness. These studies also used dining parties rather than servers as the units of analysis. Additional research involving greater numbers of servers and using servers as the units of analysis would provide more generalizable tests of server attractiveness effects.

\section{Service Quality}

Tipping is supposed to be an incentive/reward for good service (Bodvarsson and Gibson, 1994; Hemenway, 1984; Shamir, 1984). Researchers have found a positive correlation between tip sizes and service evaluations (Lynn \& Grassman, 1990; Lynn \& Graves, 1996), but this relationship accounts for only about two percent of the variability in bill-adjusted tips (Lynn, 1997). Lynn and Graves (1996) argue that this effect is too 
weak to be noticed by servers and that it calls into question the traditional role of tipping as an incentive for delivering good service. However, relationships between variables can differ at different levels of aggregation (Ostroff, 1993), so tipping could be more strongly related to service when both variables are aggregated at the server level-ofanalysis. Aggregation to the server level effectively filters out encounter level "noise" and makes it easier to detect the effects of stable server behaviors. This means that good servers may earn noticeably larger tips than do bad servers even though customers receiving good service do not leave noticeably larger tips than customers receiving bad service. This possibility needs to be tested.

\section{Self-Monitoring}

Tipping is thought to exist because the highly customized nature of many services makes it difficult for firms to monitor and control the quality of their service employees' work (Bodvarsson \& Gibson, 1994; Lynn, Zinkhan \& Harris, 1993; Shamir, 1984). Customers are in a better position to evaluate and reward a server's efforts to meet the customers' unique, individual service needs than are managers (Hemenway, 1984; Jacob $\&$ Page, 1980). This theoretical link between tipping and service customization suggests that servers who are better able to discern their customers' unique service needs and to satisfy those needs will receive larger average tips than will servers lacking these abilities. One indicator of this ability is Snyder and Gangestad's (1986) Self-Monitoring Scale, which measures individual differences in sensitivity and reactivity to cues about the situational appropriateness of a person's own behavior (Snyder, 1987). Thus, selfmonitoring may be an important determinant of differences in servers' tip incomes. This possibility has not yet been empirically tested. 


\section{$\underline{\text { Server's Sex }}$}

Tipping is a social behavior that frequently involves members of the opposite sex, so it may be affected by the dynamics of sexual attraction. Consistent with this possibility, a recent meta-analysis found that men tipped more than women, but only when the server was female (Lynn, 1997). Research outside the tipping literature has found that men favor women who are physically attractive while women favor men who have high status and wealth (Buss, 1987). These findings suggest that male customers may use tips to demonstrate their status and wealth to servers of the opposite sex more than do female customers. If so, then waitresses should receive larger average tips than do waiters. These findings also suggest that male customers may be more affected by the attractiveness of opposite sex servers than are female customers. If so, then physical attractiveness should have a stronger effect on the tip averages of waitresses than on the tip averages of waiters. One existing test of these expectations produced non-significant results (Hornik, 1993), but the relevant means were in the expected direction and additional tests may find stronger support.

\section{METHOD}

\section{$\underline{\text { Overview }}$}

Data were collected on a sample of 51 servers at a Mexican restaurant in Houston, Texas. The servers rated themselves on several service dimensions, completed Snyder and Gangestad's (1986) Self-Monitoring Scale, provided some demographic and workhistory information about themselves, and posed for individual photographs. Ten judges rated the servers' physical attractiveness from the photographs. Finally, an assistant 
manager at the restaurant recorded the charge bill and tips receipts of each server for each day and/or evening shift worked during an approximately 6 week study period. Records for two servers were incomplete and some servers worked only daytime or evening shifts, so the sample sizes reported in the results vary slightly from one analysis to another.

\section{$\underline{\text { Server Questionnaire }}$}

Each server completed a questionnaire that contained three sections relevant to this study. In the first section, servers were asked to provide information about their names, ages $(M=21.6, s d=2.66)$, sexes (27 males and 24 females $)$, ethnicities (4 Hispanics and 47 Whites), and years of experience as a waiter/waitresses $(M=2.1$, sd $=1.96$ ). In the second section, servers were asked to rate their "own strengths and weaknesses as a waiter/waitress" using a scale whose points (1-5) were labeled "Inferior," "Below Average," “Average," "Above Average," and "Superior." Servers used this scale to rate their "Attentiveness (keeping an eye on table, checking up on table, etc.)" $(M=4.13, \mathrm{sd}=.60)$, "Friendliness (smiling, chatting with customer, etc.)" $(\mathrm{M}=$ 4.33, $\mathrm{sd}=.71$ ), "Speed (promptness in greeting table, taking order, delivering food, presenting check, etc.)" $(\mathrm{M}=4.02, \mathrm{sd}=.62)$, and "Knowledge (ability to answer customers' questions about menu, food preparation, etc.)" $(M=4.25$,. $\mathrm{sd}=72)$. The ratings of Attentiveness, Speed, and Knowledge were highly correlated with one another $(.52<$ r's $<.75)$, but not with ratings of Friendliness $(.12<$ r's $<.27)$, so the three related ratings were averaged to form an index of "Service Ability" $(\mathrm{M}=4.14, \mathrm{sd}=.55$, coefficient alpha $=.83)$. In the final section of the questionnaire, servers were asked to complete Gangestad and Snyder's (1986) Self-Monitoring Scale $(\mathrm{M}=10.61, \mathrm{sd}=3.13)$. 


\section{$\underline{\text { Ratings of Attractiveness }}$}

Full figure, color photographs were taken of each server in uniform at the restaurant. Five male and five female judges independently sorted the photos into three piles, from least to most attractive. The photos of waiters and waitresses were sorted separately and each pile was allowed to contain as many or as few photos as the judge deemed appropriate. Servers were assigned 1 to 3 points reflecting each judge's evaluation of the servers' attractiveness and these points were summed across judges. Thus, our measure of server attractiveness had a minimum value of 10 , which would indicate that all ten judges put a particular server into the least attractive category, and a maximum value of 30 , which would indicate that all ten judges put a server into the most attractive category. The actual values ranged from 10 to 29 and had a mean of 18.51 (sd $=4.58)$.

\section{$\underline{\text { Charge Sales and Tip Records }}$}

Each servers' charge bill and tip receipts were recorded for every day and/or evening shift he or she worked from May 6 to June 18. The servers worked from 1 to 19 daytime shifts and worked from 2 to 23 evening shifts during this time period. The average number of daytime shifts per server was $7.67(\mathrm{sd}=5.06)$ and the average number of evening shifts per server was $10.71(\mathrm{sd}=4.67)$. These data were used to calculate the average daytime charge sales $(M=\$ 129.94, \mathrm{sd}=35.82, \mathrm{n}=46)$ and tips $(\mathrm{M}=\$ 17.71$, $\mathrm{sd}=5.55, \mathrm{n}=46)$ for each server and the average evening charge sales $(\mathrm{M}=\$ 223.38$, $\mathrm{sd}=53.30, \mathrm{n}=49)$ and tips $(\mathrm{M}=\$ 30.51, \mathrm{sd}=6.90, \mathrm{n}=49)$ for each server. 


\section{RESULTS}

Evening meals at restaurants tend to be more lengthy, formal, expensive and entertainment-oriented than are daytime meals. Thus, the determinants of servers' tip averages may be different at dinner than at lunch. Consistent with this possibility, we found only a weak relationship between the servers' average sales-adjusted tips at evening and lunch shifts, $\mathrm{r}=.26, \mathrm{n}=44, \mathrm{p}<.09$. Given this weak correlation, separate analyses were conducted on the evening and daytime tip averages. None of the independent variables other than average sales were significant predictors of daytime tip averages (all F's $<1.00$ ), so only the analyses of evening tip averages are reported here.

The servers' average evening tips were used as the dependent variable in weighted multiple regression analyses. In these analyses, each observation was weighted by the square root of the number of evening shifts on which the dependent measure was based. This weighting was used to reflect the greater reliability of tip averages based on greater numbers of work shifts.

The first analysis examined main effects by simultaneously regressing servers' evening tip averages on servers' average evening sales, physical attractiveness, self-rated service ability, self-rated friendliness, self-monitoring, sex, and number of years experience as a server (see Table 1). The full model accounted for over 90 percent of the variability in the dependent variable, $\mathrm{R}^{2}=.93$, and was highly significant, $\mathrm{F}(7,39)=$ $79.69, \mathrm{p}<.0001$. Tests of the model's parameter estimates indicated that servers received larger tip averages the larger their average sales, $t(39)=21.35$, one-tailed $p<.0001$, the more attractive they were, $\mathrm{t}(39)=1.74$, one-tailed $\mathrm{p}<.05$, the more highly they rated their service abilities, $\mathrm{t}(39)=1.77$, one-tailed $\mathrm{p}<.05$, and the higher their self-monitoring 
tendencies, $\mathrm{t}(39)=1.79$, one-tailed $\mathrm{p}<.05$. Servers' self-rated friendliness, sex and years of experience were all unrelated to their tip averages (all t's $<1.0$ ).

The second analysis examined server sex as a potential moderator of the significant main-effects in the preceding analyses. Servers' average tips were regressed on their average sales, physical attractiveness, self-rated service, self-monitoring, sex, and the products from multiplying sex with average sales, physical attractiveness, selfrated service, and self-monitoring. ${ }^{1}$ This analyses produced no significant interactions (all F's $<1.8$ ). However, statistical tests for interactions in field data are notorious for having low power (see McClelland \& Judd, 1993). Therefore, the tests for interactions reported above were supplemented with separate regressions of male and female servers' evening tip averages on the servers' average sales, physical attractiveness, self-rated service, and self-monitoring (see Table 2). These analyses indicated that physical attractiveness was a much better predictor of waitresses' average tips than of waiters' average tips (partial r's $=.40$ vs .02) and that self-rated service was a much better predictor of waiters' average tips than of waitresses' average tips (partial r's $=.49 \mathrm{vs}$ .19). ${ }^{2}$ No sizeable sex differences were observed in the predictiveness of servers' average sales (partial r's $=.98$ vs .94 ) or self-monitoring (partial $r=.28$ vs .35$)$.

\section{SUMMARY AND DISCUSSION}

This study found that attractive waitresses earned larger sales-adjusted tips than did less attractive waitresses. Attractiveness had no effect on the tips of waiters. In addition, servers who rated their own service abilities high and servers who were high in self-monitoring earned larger average sales-adjusted tips than did other servers. Server 
sex and server experience had no effects on tip earnings. Interestingly, the predictors of servers' tip earnings worked only for evening tips. None of the independent variables in this study predicted servers' average sales-adjusted lunch tips. In fact, even servers' evening tips were a weak predictor of their lunch tips. These results make several contributions of methodological, theoretic, and managerial import as described below.

\section{$\underline{\text { Methodological Contributions }}$}

The fact that the size of any particular server' tips at lunch was only weakly correlated with that server's' dinner tips affirms that the determinants of tipping in the two contexts differ. Lunch patrons are likely to take less time and perhaps to spend money in a more budget-regulated fashion than are dinner patrons and this difference may drive differences in tipping. The fact that none of the measured server differences predicted lunch tips suggests that lunch tips are relatively insensitive to differences in server conduct or attributes. Perhaps differences in lunch tipping can be better predicted by demographic differences between patrons. These considerations are of methodological importance to tipping researchers. They suggest that (a) lunch tips should be separated out from combined lunch/dinner data sets if the goal is to examine the impact of server behavior or attributes on tips, and (b) lunch tipping should be studied in its own right, possibly as an outcome of patron differences.

A second methodological contribution of our study derives from its design in terms of level-of-analysis and the related issue of server sample size. Prior studies of tipping behavior have focused exclusively on encounter-level differences in tip amounts - i.e., the customer-server encounter was used as the unit of analysis. Given that sex, physical attractiveness, service ability, and self-monitoring are all server-level variables 
rather than encounter-level variables, examination of the effects of these predictors was both more appropriate and more powerful at the server level. Analysis at the server level effectively aggregates service encounters to allow a more accurate assessment of the impact of server behaviors and attributes that remain relatively stable within each server. Further, our sample size of 51 servers is over three times as large as that of any other study in this area (see May, 1978, who studied 16 servers) and is five times larger than any published study of server sex differences (see Hornik, 1993, who studied 8 servers). The larger sample size of servers in our study allows a more confident assessment of the impact of server-level differences on tipping.

\section{Theoretical Contributions}

This work makes three substantive contributions to the literature on tipping. The first theoretical or substantive contribution of this work emerges from the detection of a service ability effect on servers' average sales-adjusted tips. Previous studies have found only very weak service effects on tipping of around $r=.12$ (Lynn, 1997), while the present study finds a stronger effect of $r=.27$-- a fivefold increase in explained variance. For waiters, service ability had an even stronger effect of $r=.49$. The comparatively strong service-tipping relationship observed in this study may be attributable to our examination of tipping at the server level-of-analysis in contrast to previous studies that examined tipping at the encounter level. Aggregating tips across encounters to obtain server averages reduces the variance in the dependent measure that is not attributable to server characteristics. This reduction in error variance increases the relative size of server effects and makes them easier to detect. The increased magnitude of the service-tipping relationship at the server level-of-analysis is important because it 
means that servers (especially male servers) are more likely to notice the relationship than had previously been asserted (Lynn \& Graves, 1996). Thus, our results help justify the perception of tips as an economic incentive (Bodvarsson \& Gibson, 1994; Hemenway, 1984; Jacob \& Page, 1980).

There are two plausible explanations for the relationship we found between selfrated service ability and tipping. First, servers who earned larger sales-adjusted tips may have interpreted this outcome as an indication of their own service abilities and may have rated themselves accordingly. Second, servers' who rated their service abilities highly may have received larger tips because consumers rewarded them for delivering better service. Although both of these explanations are plausible, the second explanation is more parsimonious than the first when other tipping research is taken into account. Lynn and Grassman (1990), Lynn and Graves (1996) and May (1978) found that restaurant tips were related (though weakly) to the quantity and/or quality of service at the serviceencounter level-of-analysis while Fitzsimmons and Mauer (1991) found that tips were related to server attentiveness at the restaurant level-of-analysis. The idea that consumers reward better service with larger tips is unique in being able to explain all of these tipping-service relationships.

A second theoretical contribution of this research is the observed effect of the self-monitoring server characteristic on sales-adjusted tip averages. High self-monitors received larger tips than did low self-monitors. This finding is consistent with economic theories linking tipping with service customization (Bodvarsson \& Gibson, 1994; Hemenway, 1984; Jacob \& Page, 1980), because high self-monitors should be more 
sensitive to population and individual differences among their customers and should be more inclined to adjust their service delivery accordingly.

A third theoretical contribution of this study is the physical attractiveness effect on tips, which was carried by female servers. Although the interaction effect between sex of server and physical attractiveness was not statistically significant, attractiveness accounted for substantially greater variability in the sales-adjusted tip averages of waitresses than of waiters (partial r's $=.40 \mathrm{vs} .02$ ). This result might have emerged from a higher societal value placed on physical attractiveness for females than for males. An alternative explanation is that male patrons' tipping behavior is more strongly influenced by their sexual response to opposite-sex servers. Both of these explanations are congruent with stereotypic North American sex role norms. However, the present study lacks information on the sex and tip size of individual patrons and is thus unable to distinguish between the two proposed accounts. We anticipate that future studies will further illuminate this mechanism.

\section{Managerial Implications}

In addition to its methodological and theoretical contributions, this study has considerable practical relevance to restaurant managers and servers. In this sample, the highest tipped employees were beautiful females, highly competent males and high selfmonitors of both sexes. Servers lacking these attributes may want to consider other lines of work, particularly if they are concerned about their earnings relative to those of coworkers. Similarly, managers might want to consider these attributes when making hiring decisions. While ethical and legal concerns strongly discourage the application of different hiring criteria to male and female servers, there are no legal problems with 
using attractiveness, service ability and/or self-monitoring as hiring criteria for servers of both sexes. To the extent that better-compensated servers might be more satisfied and more loyal (Lynn, 1996), employers could consider our results as evidence in support of such hiring criteria. With this in mind, it is worth noting that our failure to find a relationship between tip earnings and years of service experience suggests that previous work experience is a poor indicator of service ability. We recommend the use of a systematic interview (see Simons, 1995), followed by a few hours observing compensated serving work, to assess this hiring criterion.

\section{$\underline{\text { Study Limitations }}$}

As with any study, this one has methodological attributes that limit the confidence with which one can interpret its results. First, the study is correlational in design, which means that it cannot justify strong causal claims. However, server characteristics such as sex and self-monitoring cannot be manipulated and the experimental manipulation of server attractiveness in real-world restaurant settings is virtually impossible, so correlational study appears to be the only possible avenue of approach. Second, the study used self-assessments of service quality, which might be influenced by self-serving biases. However, the relationship of these self-assessments to tipping provides some evidence for their validity. Furthermore, a meta-analysis of research on salesperson effectiveness has shown that there is little difference in the predictors of managerassessed and self-assessed performance (Ford, Walker, Churchill \& Hartley, 1987), suggesting that self-assessments can be valid measures of performance. Finally, the study examined tipping at a single mid-scale restaurant in Texas, which limits the generalizability of our findings. It is possible that the determinants of tipping will be 
influenced by the luxury level of the restaurant, the extent to which it draws on repeat business, it's geographic location and other restaurant-related factors (see Lynn, 1997). These issues warrant further exploration. Nevertheless, the present study provides a reasonable and provocative contribution to our understanding of restaurant tipping. 


\section{REFERENCES}

Aiken, L.S. \& West, S.G. (1991). Multiple regression: Testing and interpreting interactions. Newbury Park, CA: Sage.

Bodvarsson, O.B. \& Gibson, W.A. (1994). Gratuities and customer appraisal of service: Evidence from Minnesota restaurants. Journal of Socio-Economics, 23, 287-302.

Buss, D.M. (1987). Sex differences in human mate selection crietia: An evolutionary perspective. In C. Crawford, M. Smith, \& D. Krebs (Eds.), Sociobiology and psychology: Issues, ideas, and findings. Hillsdale, NJ: Erlbaum.

Cohen, J. \& Cohen, P. (1975). Applied multiple regression/correlation analysis for the behavioral sciences. Hillsdale, NJ: Earlbaum.

Crespi, L.P. (1947). The implications of tipping in America. The Public Opinion Quarterly, 11, 424-435.

Fitzsimmons, J. A. \& Maurer, G. B. (1991). A walk-through audit to improve restaurant performance. Cornell H.R.A. Quarterly, 31, 95-99.

Ford, N.M., Walker, O.C., Churchhill, G.A. \& Hartley, S.W. (1987). Selecting successful salespeople: A meta-analysis of biographical and psychological selection criteria. Review of Marketing, $\underline{X}, 90-131$.

Hemenway, D. (1984). Prices and choices: Micro-economic vignettes. Cambridge, MA: Ballinger Publishing Co.

Hornik, J. (1993). Tactile stimulation and consumer response. Journal of Consumer Research, 19, 449-458.

Jacob, N. \& Page, A. (1980). Production, information costs, and economic organization: The buyer monitoring case. American Economic Review, 70, 476-478. 
Lynn, M. (1996). Seven ways to increase servers' tips. Cornell H.R.A. Quarterly, 37, 24-29.

Lynn, M. (1997). The determinants of restaurant tipping: A meta-analytic review.

Annual Conference of the Society for the Advanement of Behavioral Economics,

Lexington, VA, June.

Lynn, M. \& Grassman, A. (1990). Restaurant tipping: An examination of three

'rational' explanations'. Journal of Economic Psychology, 11, 169-181.

Lynn, M. \& Graves, J. (1996). Tipping: An incentive/reward for service?

Hospitality Research Journal, 20, 1-14.

Lynn, M., Zinkhan, G.M. \& Harris, J. (1993). Consumer tipping: A cross-country study. Journal of Consumer Research, 20, 478-488.

May, J.M. (1978). Tip or treat: A study of factors affecting tipping behavior.

Unpublished master's thesis, Loyola University of Chicago.

McClelland, G.H. \& Judd, C.M. (1993). Statistical difficulties of detecting interactions and moderator effects. Psychological Bulletin, 114, 376-390.

O'Connor, C.M. (1971). Wages and tips in restaurants and hotels. Monthly Labor Review, $94,47-51$.

Ostroff, C. (1993). Comparing correlations based on individual-level and aggregated data. Journal of Applied Psychology, $\underline{78}$, 569-582.

Patzer, G.L. (1985). The Physical Attractiveness Phenomena. New York: Plenum Press.

Shamir, B. (1984). Between gratitude and gratuity: An analysis of tipping. Annals of Tourism Research, 11, 59-78.

Simons, T. (1995). Interviewing job applicants: How to get beyond first impressions. Cornell H.R.A. Quarterly, $\underline{36}$, 21-27. 
Snyder, M. (1987). Public appearances, private realities: The psychology of selfmonitoring. New York: W.H. Freeman and Co.

Snyder, M. \& Gangestad, S. (1986). On the nature of self-monitoring: Matters of assessment, matters of validity. Journal of Personality and Social Psychology, 51, 125-139. 


\section{ENDNOTES}

${ }^{1}$ Testing for interactions is a powerful way to explore the causal mechanisms that undergird empirical relationships between variables. A significant interaction term changes the slope of an empirical regression line, and so indicates that the relationship between two variables is, in turn, dependent on a third variable. In the present study, the interactions were between continuous variables (average sales, physical attractiveness, self-rated service, self-monitoring) and a dichotomous variable (sex). As such, the multiplicative interaction terms in the regression did not suffer from the multicollinearity and interpretation problems that can complicate tests of interaction terms among continuous variables. Aiken and West (1991) describe a method for testing interactions among continuous variables. By subtracting the mean variable value from all continuous variables prior to the generation of interaction terms, multicollinearity between variables and their interaction terms can be eliminated, and the resulting coefficients can readily be translated into interpretable slopes. These procedures are unnecessary when one of the elements of the interaction is dichotomous, as the resulting B-coefficient can simply be added for the group coded as " 1. "

${ }^{2}$ Partial r's are correlations between two variables after statistically controlling for the effects of one or more covariates on both of the correlated variables (see Cohen and Cohen, 1975). Partial r's share significance tests with the corresponding parameter estimates from multiple regression models. In other words, the t-test of a B-coefficient from a multiple regression model also tests the significance of the corresponding partial correlation - the correlation between the D.V. and the corresponding I.V. from that model when all the other I.V. s are held constant. 
TABLE 1. Results of a simultaneous multiple regression analysis predicting servers' average evening tips.

\begin{tabular}{lcccc}
\hline Source & Partial r & df & F-value & p-value \\
\hline \hline & & & & \\
Model & & 7 & 79.69 & .0001 \\
Average Sales & .96 & 1 & 455.79 & .0001 \\
Physical Attractiveness & .27 & 1 & 3.03 & .090 \\
Service Ability & .27 & 1 & 3.14 & .085 \\
Friendliness & .10 & 1 & 0.42 & .520 \\
Self-Monitoring & .28 & 1 & 3.22 & .081 \\
Sex & .12 & 1 & 0.53 & .473 \\
Service Experience & .02 & 1 & 0.02 & .878 \\
Error & & 39 & $(10.00)$ & \\
& & & & \\
\hline
\end{tabular}

Note - The number in parentheses is the mean square error. T-tests of the corresponding parameter estimates from this regression model can be obtained by taking the square root of the F-values. One-tailed p-values for those t-tests can be obtained by halving the pvalues reported for the F-tests. 
TABLE 2. Results of simultaneous multiple regression analyses predicting average evening tips for waiters and waitresses.

\begin{tabular}{|c|c|c|c|c|}
\hline Sample/Source & Partial r & $\mathrm{df}$ & F-value & p-value \\
\hline \multicolumn{5}{|l|}{ WAITERS } \\
\hline Model & & 4 & 123.25 & .0001 \\
\hline Average Sales & .98 & 1 & 459.48 & .0001 \\
\hline Physical Attractiveness & .02 & 1 & 0.01 & .90 \\
\hline Service Ability & .49 & 1 & 6.23 & .02 \\
\hline Self-Monitoring & .28 & 1 & 1.68 & .21 \\
\hline Error & & 20 & $(7.33)$ & \\
\hline \multicolumn{5}{|l|}{ WAITRESSES } \\
\hline Model & & 4 & 38.11 & .0001 \\
\hline Average Sales & .94 & 1 & 119.05 & .0001 \\
\hline Physical Attractiveness & .40 & 1 & 3.16 & .09 \\
\hline Service Ability & .19 & 1 & 0.61 & .44 \\
\hline Self-Monitoring & .35 & 1 & 2.42 & .14 \\
\hline Error & & 17 & $(13.01)$ & \\
\hline
\end{tabular}

Note - Numbers in parentheses are mean square errors. T-tests of the corresponding parameter estimates from these regression models can be obtained by taking the square root of the F-values. One-tailed p-values for those t-tests can be obtained by halving the p-values reported for the F-tests. 